\title{
ON THE RELATIVE VITALITY OF THE SEXES.*
}

To the Couneil of the Statistical Society.

My LoRDs AND GENTLEmEn,-The Registrar-General having advised me to render public a table deduced from his 14 th and 15 th Annual Reports (1851-2), published this year, and whereof he has faroured me with a copy, I beg to communicate it herewith to your Society.

In the 14th (1851) Report are two companion tables (Table XII., p. 16), and in the 15th (1852) are similar tables (Table XI., p. 9), showing the per centage of mortality in each sex to the numbers living at that age in various years. Thus in the year 1838 , of 100,000

$\left.\begin{array}{l}\text { males } \\ \text { females }\end{array}\right\}$ then living, aged from 25 to 35 , there died $\left\{\begin{array}{l}1,064 \\ 1,046 .\end{array}\right.$

It occurred to me, that if the quotient of these two numbers $\frac{1.064}{1.046}$ $=1.017$, were tabulated, it would nearly represent the law of mortality between the two sexes, independent of epidemics and other aggravating causes. Thus, if 10 men and 9 women, 20 men and 18 women, 50 men and 45 women, \&c., died per 10,000 of the then existing population, the proportion of 10 to 9 would still remain unaltered.

These quotients are exhibited in the annexed table (see next page).

Thus, of a population of 100,000 of each sex between 35 and 45 years of age, in the year 1849, there died 978 males to every 1,000 females; in $1851,1,036$ males to 1,000 females, \&c.

The last column shows how small are the extreme variations for the period 1838-52, or half a generation.

The table indicates that man is relatively weaker than woman at 0-5

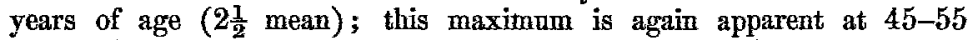
(i. e., 50); they are nearly equal at $5-10\left(7 \frac{1}{2}\right), 35-45(40)$; and at $25-35$ (30), the principal period of reproduction, females are less able to resist death, in the proportion of 95 to 100 . The general regularity of this minimum at this age is a most striking feature of the table: and if the birth-register would indicate the age of the mother and the rank of the child (as 1st, 2nd, 5th, \&c.), we should bave a still better elucidation of this FACT.

The irregularity in very aged persons, of 95 and upwards, is of no great moment, considering the smallness of the data, the occasional impossibility of accurately knowing the exact age of these time-honoured individuals, \&c.

The general agreement of the two penultimate columns makes me suspect some little misprint in the Registrar's numbers for the ages 25-35, $35-45$.

If the report-numbers could be ascertained for every single year of life, we might thence deduce the numbers for every single year of life, and perhaps light on the mathematical law of this portion of vital statistics.

I am, \&c.

S. M. DRACH,

23, Walpole Street, Ohelsea, (F.R.A. \& R.G.S. 10th December, 1855.

* Extrasted from the Journal of the Statistical Society, June, 1856. 


\begin{tabular}{|c|c|c|c|c|c|c|c|c|c|c|c|c|c|}
\hline 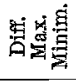 & 骂 & $\stackrel{-10}{\%}$ & $\stackrel{?}{\stackrel{P}{\varphi}}$ & $\stackrel{1}{9}$ & 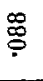 & $\stackrel{\vec{S}}{\rightarrow}$ & 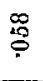 & $\stackrel{\wp}{\varnothing}$ & ஜ̊: & $\stackrel{\infty}{\mathscr{\varphi}}$ & ఫ্t & $\stackrel{\circ}{9}$ & $\vec{q}$ \\
\hline 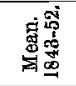 & 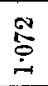 & $\begin{array}{l}\mathscr{E} \\
\stackrel{-}{二}\end{array}$ & $\stackrel{\infty}{\stackrel{\infty}{\rho}}$ & 品 & \begin{tabular}{l}
20 \\
\hdashline \\
$:$
\end{tabular} & \begin{tabular}{l}
$\stackrel{\infty}{0}$ \\
\hdashline \\
\hdashline
\end{tabular} & $\stackrel{\infty}{\mathscr{g}}$ & 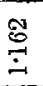 & $\underset{\Xi}{\stackrel{\Xi}{\Xi}}$ & $\underset{\dot{m}}{\stackrel{8}{+}}$ & $\overrightarrow{\stackrel{9}{\ominus}}$ & $\begin{array}{l}\mathscr{0} \\
\stackrel{\leftrightarrow}{\circ}\end{array}$ & 祭 \\
\hline 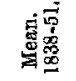 & $\stackrel{10}{\circ}$ & $\stackrel{\Im}{\mathscr{\Xi}}$ & $\stackrel{\overbrace{}}{\stackrel{0}{0}}$ & $\vec{\varphi}$ & $\begin{array}{l}\mathscr{O} \\
\%\end{array}$ & $\frac{9}{5}$ & $\begin{array}{l}\stackrel{2}{2} \\
:\end{array}$ & $\stackrel{10}{\vec{H}}$ & 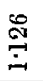 & $\frac{20}{\Xi}$ & $\begin{array}{l}\stackrel{0}{\circ} \\
\stackrel{\leftrightarrow}{\dot{H}}\end{array}$ & $\begin{array}{l}\mathscr{\mathscr { g }} \\
\stackrel{\mathscr{g}}{\mathscr{g}}\end{array}$ & 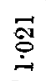 \\
\hline 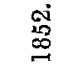 & $\stackrel{0}{5}$ & 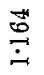 & 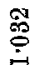 & 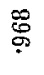 & $\begin{array}{l}\infty \\
00 \\
0 \\
0\end{array}$ & $\begin{array}{l}\text { g } \\
\stackrel{5}{\$}\end{array}$ & $\stackrel{\infty}{\stackrel{\rho}{ٍ}}$ & $\stackrel{g}{\stackrel{g}{\Xi}}$ & $\stackrel{40}{ \pm 0}$ & 宫 & $\stackrel{0}{5}$ & ஜ્ळ & 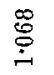 \\
\hline$\underset{\infty}{2}$ & 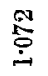 & 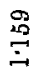 & $\stackrel{9}{\stackrel{9}{9}}$ & 命 & 总 & $\frac{\Re}{\not+p}$ & 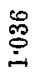 & $\stackrel{0}{\underline{1}}$ & $\begin{array}{l}\stackrel{\infty}{\infty} \\
\stackrel{\oplus}{-}\end{array}$ & 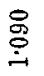 & 홍 & 穷 & $\begin{array}{l}\text { ণ } \\
\text { क़े }\end{array}$ \\
\hline 离 & 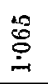 & 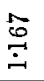 & 号 & 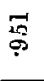 & 恕 & $\begin{array}{l}\stackrel{8}{\circ} \\
\stackrel{0}{\circ}\end{array}$ & চ্ & $\stackrel{\bullet}{\Xi}$ & 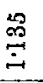 & $\stackrel{\infty}{\stackrel{D}{\Xi}}$ & $\stackrel{20}{\stackrel{2}{\leftrightarrows}}$ & 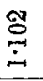 & 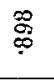 \\
\hline 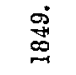 & $\begin{array}{l}10 \\
0 \\
9 \\
\end{array}$ & 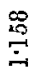 & $\stackrel{9}{9}$ & 文 & $\begin{array}{l}\text { मे } \\
\text { के }\end{array}$ & 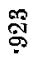 & $\begin{array}{l}\infty \\
\frac{1}{0}\end{array}$ & 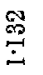 & 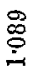 & $\begin{array}{l}\stackrel{0}{0} \\
\stackrel{9}{9}\end{array}$ & 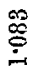 & $\begin{array}{l}\stackrel{8}{8} \\
\stackrel{8}{\circ}\end{array}$ & : \\
\hline $\begin{array}{l}\infty \\
\stackrel{\infty}{*} \\
\stackrel{*}{*}\end{array}$ & $\stackrel{+}{\stackrel{5}{\circ}}$ & $\begin{array}{l}\stackrel{\mathscr{C}}{\leftrightarrows} \\
\stackrel{\sim}{\sim}\end{array}$ & $\begin{array}{l}\stackrel{0}{*} \\
\stackrel{f}{\circ}\end{array}$ & क् & 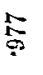 & $\overrightarrow{\vec{H}}$ & $\stackrel{\text { gి }}{\stackrel{9}{\circ}}$ & 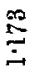 & 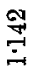 & $\stackrel{\stackrel{\sigma}{\rightrightarrows}}{\underset{\sim}{\leftrightarrows}}$ & 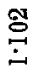 & $\begin{array}{l}\stackrel{9}{\circ} \\
\stackrel{9}{*}\end{array}$ & 乌ั \\
\hline$\underset{\sim}{\stackrel{5}{*}}$ & $\stackrel{?}{\stackrel{2}{\circ}}$ & $\begin{array}{l}\infty \\
\stackrel{\infty}{20} \\
\stackrel{5}{ \pm}\end{array}$ & 尽 & 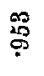 & $\bar{\rho}$ & $\begin{array}{l}\text { 品 } \\
\text { क्र }\end{array}$ & 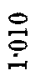 & 焉 & $\overrightarrow{\vec{\sigma}}$ & $\begin{array}{l}\text { 骂 } \\
\stackrel{9}{-1}\end{array}$ & $\begin{array}{l}\widehat{5} \\
\stackrel{9}{\circ}\end{array}$ & 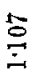 & 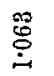 \\
\hline 兽 & 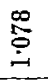 & $\stackrel{\Re}{Ð}$ & $\stackrel{\Xi}{\stackrel{\circ}{\leftrightarrows}}$ & $\frac{50}{9}$ & \begin{tabular}{l}
\multicolumn{2}{c}{} \\
9 \\
9
\end{tabular} & $\frac{8}{6}$ & 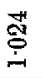 & 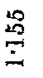 & 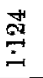 & $\begin{array}{l}\stackrel{\infty}{\mathscr{O}} \\
\stackrel{\mathscr{D}}{ }\end{array}$ & $\stackrel{\text { }}{\stackrel{\leftrightarrow}{\circ}}$ & $\overrightarrow{\stackrel{\leftrightarrow}{\dot{\theta}}}$ & $\begin{array}{l}\mathscr{Q} \\
\stackrel{8}{6}\end{array}$ \\
\hline & $\stackrel{2}{\stackrel{2}{5}}$ & $\underset{⿱ 乛}{\stackrel{2}{2}}$ & 总 & $\frac{R}{\sigma}$ & $\begin{array}{l}\mathscr{6} \\
\stackrel{6}{\circ}\end{array}$ & \begin{tabular}{l}
29 \\
\multirow{9}{*}{}
\end{tabular} & 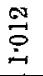 & $\stackrel{g}{\stackrel{\Xi}{\Xi}}$ & $\stackrel{20}{\stackrel{2}{-}}$ & 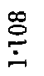 & $\stackrel{\leftrightarrow}{9}$ & $\begin{array}{l}20 \\
\stackrel{9}{9}\end{array}$ & $\underset{\stackrel{\leftrightarrow}{0}}{\stackrel{\pi}{0}}$ \\
\hline 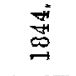 & 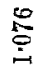 & 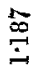 & హౌ & $\begin{array}{l}\text { 앙 } \\
\text { क }\end{array}$ & \begin{tabular}{l}
0 \\
\multirow{0}{*}{} \\
0
\end{tabular} & $\begin{array}{l}\text { \#े } \\
\text { के }\end{array}$ & 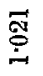 & $\stackrel{\text { 趈 }}{\stackrel{-}{-}}$ & 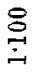 & 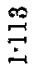 & 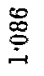 & 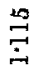 & $\begin{array}{l}8 \\
\stackrel{8}{6}\end{array}$ \\
\hline 离 & $\stackrel{\circ}{5}$ & $\stackrel{ }{ }$ & $\begin{array}{l}\text { कू } \\
\text { क्ष }\end{array}$ & 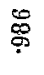 & $\begin{array}{l}10 \\
0 \\
0 \\
0\end{array}$ & T) & $\begin{array}{l}0 \\
\%\end{array}$ & $\stackrel{8}{\Xi}$ & $\stackrel{\Sigma}{\Xi}$ & $\stackrel{\stackrel{\vartheta}{\rightrightarrows}}{\underset{\Xi}{*}}$ & $\overrightarrow{\stackrel{\rho}{ٍ}}$ & 옹 & $\frac{R}{\sigma}$ \\
\hline$\underset{\infty}{\stackrel{\sigma}{*}}$ & $\begin{array}{l}\infty \\
\stackrel{8}{\circ}\end{array}$ & $\stackrel{R}{I}$ & $\frac{\vec{t}}{\frac{\sigma}{0}}$ & $\frac{R}{Q}$ & 品 & $\begin{array}{c}\text { શิ } \\
\text { क़ }\end{array}$ & $\vec{g}$ & $\stackrel{\mathscr{m}}{\Xi}$ & $\stackrel{\infty}{\stackrel{9}{\Xi}}$ & $\stackrel{\widehat{9}}{9}$ & $\underset{\exists}{\stackrel{\Xi}{\Xi}}$ & $\begin{array}{l}\stackrel{40}{0} \\
\stackrel{0}{0}\end{array}$ & 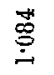 \\
\hline 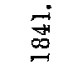 & 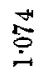 & $\stackrel{\mathscr{O}}{\Xi}$ & 埋 & 官 & $\stackrel{\mathscr{0}}{\circ}$ & 중 & 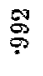 & $\stackrel{\infty}{\stackrel{0}{ \pm}}$ & $\stackrel{20}{\overrightarrow{2}}$ & $\underset{ت}{\stackrel{g}{\rightleftarrows}}$ & $\begin{array}{l}\stackrel{\leftrightarrow}{\circ} \\
\stackrel{\leftrightarrow}{-}\end{array}$ & $\frac{9}{5}$ & 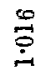 \\
\hline 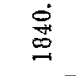 & 5 & $\stackrel{2}{\Sigma}$ & 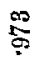 & $\begin{array}{l}\infty \\
\stackrel{0}{0}\end{array}$ & $\begin{array}{l}\stackrel{8}{\circ} \\
: 0\end{array}$ & $\begin{array}{l}\mathscr{P} \\
\text { }\end{array}$ & $\begin{array}{l}\stackrel{2}{2} \\
\stackrel{9}{\%}\end{array}$ & $\stackrel{9}{\exists}$ & $\Xi$ & $\frac{20}{2}$ & $\stackrel{?}{\stackrel{R}{\rho}}$ & $\stackrel{8}{9}$ & 알 \\
\hline $\begin{array}{l}\text { 吕 } \\
\text { 怘 }\end{array}$ & $\begin{array}{l}\mathscr{0} \\
\stackrel{0}{0}\end{array}$ & $\begin{array}{l}\stackrel{8}{0} \\
\stackrel{4}{4}\end{array}$ & $\begin{array}{l}5 \\
6\end{array}$ & $\begin{array}{l}10 \\
9\end{array}$ & 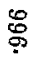 & $\frac{\Re}{\sigma}$ & $\stackrel{28}{\stackrel{2}{0}}$ & $\overrightarrow{0}$ & $\stackrel{8}{\stackrel{8}{2}}$ & 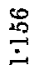 & $\stackrel{\infty}{\stackrel{2}{\rightleftarrows}}$ & $\frac{8}{2}$ & 吕 \\
\hline 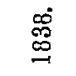 & $\stackrel{20}{9}$ & 芯 & $\stackrel{5}{\circ}$ & $\begin{array}{l}0 \\
0 \\
0\end{array}$ & 章 & 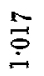 & 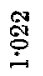 & 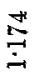 & $\stackrel{\stackrel{20}{*}}{\stackrel{9}{ت}}$ & $\begin{array}{l}\stackrel{\infty}{6} \\
\stackrel{\sim}{*}\end{array}$ & $\vec{\exists}$ & 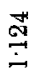 & 宫 \\
\hline 焉 & 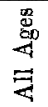 & $\frac{1}{6}$ & $\frac{0}{11}$ & $\stackrel{10}{1}$ & ${ }_{2}^{00}$ & $1_{20}^{20}$ & 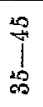 & 10 & $\prod_{20}^{20}$ & 10 & $\prod_{20}^{\infty}$ & $\prod_{\infty}^{20}$ & 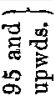 \\
\hline
\end{tabular}

